\title{
Ability of Dental Students to Deliver Oxygen in a Medical Emergency
}

\author{
Thao T. Le, B.S., D.D.S.; Erica L. Scheller, B.S.; Harold M. Pinsky, D.D.S.; \\ Stephen J. Stefanac, D.D.S., M.S.; Russell S. Taichman, D.M.D., D.M.Sc.
}

Abstract: This study was conducted to evaluate the ability of dental students to administer emergency oxygen to a patient during a simulated emergency. Forty third-year (D3) and fourth-year (D4) dental students were recruited and asked to demonstrate their ability in managing a simulated angina attack. Students were tested on their knowledge related to emergency medical protocols, the time taken to obtain oxygen, and operation of the oxygen equipment. Of the subjects tested, 68 percent independently identified the need for oxygen and the correct location of the equipment in the dental school. Only 15 percent of the students completed the experiment within a predetermined optimal time frame, and 50 percent of all students did not successfully operate the tank regulator to administer oxygen correctly. Although most participants in the study were able to verbalize the proper protocol for managing medical emergencies, the chairside execution in this situation demonstrates room for improvement. Incorporation of periodic simulation exercises, in addition to classroom education, is likely to improve the ability of dental students to manage medical emergencies.

Dr. Le is a graduate of the Scholars Program in Dental Leadership, School of Dentistry, University of Michigan and is currently in the Oral \& Maxillofacial Surgery Residency Program, Emory University; Ms. Scheller is a student in the Oral Health Sciences Ph.D. Program, School of Dentistry, University of Michigan; Dr. Pinsky is in private practice and is in the Scholars Program in Dental Leadership, School of Dentistry, University of Michigan; Dr. Stefanac is Associate Dean for Clinical Affairs, Department of Periodontics and Oral Medicine, School of Dentistry, University of Michigan and in the school's Scholars Program in Dental Leadership; and Dr. Taichman is Director of the University of Michigan School of Dentistry's Scholars Program in Dental Leadership. Dr. Le and Dr. Scheller contributed equally to this study. Direct correspondence and requests for reprints to Dr. Russell S. Taichman, Department of Periodontics and Oral Medicine, School of Dentistry, University of Michigan, 1011 North University Avenue, Ann Arbor, MI 48109-1078; 734-764-9952 phone; 734-763-5503 fax; rtaich@umich.edu.

Key words: dental education, medical emergencies, oxygen, simulation, dental students

Submitted for publication 7/11/08; accepted 1/26/09

A s members of a health care team, dental professionals must be competent in managing medical emergencies that arise within their practice settings. The likelihood that a dentist will face an emergency situation during his or her career is high; a study in 2005 revealed that close to 60 percent of dentists had participated in the management of one to three medical emergencies annually. ${ }^{1}$ A survey of 4,039 private dentists in the United States and seven Canadian provinces in 1992 found that over 30,000 medical emergencies had occurred in their practices during a ten-year period. ${ }^{2}$ Currently, there are more than 37 million people in the United States over the age of sixty-five, and this number is expected to rise to 70 million by $2030 .{ }^{3}$ Therefore, as the population ages and presents with multiple chronic systemic diseases, the incidence of medical emergencies is likely to increase.

One of the most important medications used during medical emergencies is oxygen. Oxygen should be administered to people experiencing cardiovascular emergencies, noncardiovascular stressrelated emergencies such as seizures and thyroid crises, and noncardiovascular non-stress-related emergencies such as allergies and orthostatic hypotension. ${ }^{4}$ When used correctly, oxygen is useful in managing many life-threatening events. For example, ischemic periods lasting more than ten seconds may lead to seizures; those lasting longer than ten minutes are likely to result in permanent brain damage. ${ }^{5}$ In fact, oxygen administration is recommended for almost all medical emergencies except during hyperventilation..$^{6,7}$ All dental professionals must be able to correctly administer oxygen, at therapeutic levels, during in-office emergencies.

Dental school curricula are guided by standards developed by the Commission on Dental Accreditation (CODA). ${ }^{8}$ One CODA educational standard states that "Graduates must be competent in providing appropriate life support measures for medical emergencies that may be encountered in dental practice." Another standard regarding patient care requires that "All students, faculty, and support staff involved in the direct provision of patient care must be competent in the delivery of basic life support (B.L.S.), including cardiopulmonary resuscitation, 
and the ability to manage common medical emergencies." Although clinical emergency training is provided in all U.S. dental schools, it is necessary that these skills be quantitatively assessed. In fact, a recent survey among dental students overwhelmingly identified that most graduating dental students believed that emergency medical training should be increased and periodically reassessed during their dental curricula. ${ }^{10}$ A survey conducted by the American Dental Education Association (ADEA) found that between 16 and 17 percent of these graduates felt that inadequate educational time was devoted to emergency medical training. ${ }^{11}$ Each aspect of dental education - specifically, in this case, management of a medical emergency - has to be regularly updated to be effective and relevant for today's student. ${ }^{10}$

The purpose of this study was to determine the effectiveness of one educational program in preparing its students to manage and deliver oxygen in a simulated medical emergency in a clinical setting. Our specific aim was to examine dental students' ability to properly administer oxygen to a simulated patient. To this end, we evaluated the ability of dental students to recognize the necessity to administer oxygen in an emergency situation; locate and retrieve the oxygen from a centralized location; and correctly operate and administer oxygen to a simulated patient. We hypothesized that dental students in our program would be able to correctly identify the medical situation and administer therapeutic oxygen levels appropriately based on the training they had received during the regular curriculum.

\section{Materials and Methods}

Approval was obtained from the University of Michigan Institutional Review Board prior to initiation of the study. Twenty subjects were recruited using convenience sampling from each of the thirdand fourth-year (D3 and D4) dental classes at the University of Michigan School of Dentistry. Each class is comprised of approximately 110 students. Two of the investigators (TTL and ELS) solicited volunteers by approaching potential subjects who were not actively engaged in patient care. During any three-hour clinic session, there were approximately thirty students present in each of four undergraduate clinics. Each participant completed a written consent form before participating in the study.

Subjects were brought to a designated examination area within one of the clinics that contained a mannequin head in a dental chair. The case scenario was initiated by handing the subject the first of three cards (Table 1), which described a potential emergency situation. The first card (Card \#1) asked the subject to provide a free-form verbal response outlining a plan of action. If the subject identified the need to administer oxygen, a second card (Card \#2) was provided, instructing the subject to obtain and deliver oxygen to the mannequin. In the event that the subject was unable to identify the need to administer oxygen within two and a half minutes, a third card was provided (Card \#3), which asked the subject whether anything else could be done to

Table 1. Text on emergency scenario cards presented to subjects in the study

Emergency Scenario (Card \#1)

You are currently in the middle of extracting a tooth on this 62-year-old male. His medical history is significant for obesity, angina pectoris, cardiovascular disease, and hypertension. You have been working for about 30 minutes, and the tooth suddenly breaks in half with a very loud cracking sound. This startles the patient, who was already apprehensive because of the nature and length of the procedure. He suddenly sits forward and presses his fist to his chest. You ask if he is ok, and he gasps that he has a burning, pressing sensation. He uses his other hand to reach into his pocket to pull out a small bottle. He opens the bottle and takes one small pill. You are his primary provider. Please treat this as a true emergency and verbalize to the investigators what you will do to address the situation.

Acquisition of Oxygen (Card \#2)

Treat this situation as a true emergency. Obtain the oxygen tank, and return to administer it to the patient immediately. Do not speak to the co-investigators until you feel that you have completely finished the exercise. Return this card to the co-investigators when you are ready to start.

Completion of the Experiment (Card \#3)

Thank you for your response. Is there anything else that can be done to address this situation? 
address the situation. If subjects failed to verbalize the need to administer oxygen during the scenario in response to Cards \#1 or \#3, they were asked to follow the directives provided on Card \#2. A conscious effort was made to solicit an equal number of D3 and D4 dental students and to limit the time that the clinical scenario was taking place in each of the four clinics to minimize discussion among potential subjects.

The parameters evaluated during the scenario were the following: 1) the time necessary to identify the need for oxygen; 2) the ability to locate the oxygen tank in the clinic and bring it to the examination area; and 3) the ability to operate the tank and deliver oxygen at therapeutic concentration. Two investigators were present in the cubicle at the start of the experiment (TTL and ELS). When the subject left the cubicle, one investigator followed the subject to observe and record his or her actions, and the second investigator remained in the cubicle to record the time to complete the task (Figure 1). Time was recorded in minutes and seconds using a stopwatch, on forms designed for the study. The session was considered complete when the subject stated that he or she was finished operating the oxygen tank. At that time, the flow rate set on the oxygen regulator was recorded. At the completion of the clinical session, a written survey was handed to all study participants requesting their opinions regarding the curriculum at the School of Dentistry for managing medical emergencies.

At the University of Michigan School of Dentistry, the emergency oxygen tanks are located at a centralized dispensing desk where students obtain instruments and materials for patient care (Figure 2). Dispensing desks are located approximately twenty-five feet from the entrances to each of the four clinics, each of which is sixty to seventy feet in length. The most remote treatment areas from the dispensing desks were purposefully chosen for this study, so we could evaluate the minimum time required to obtain the oxygen equipment. The time the subject would need to travel to reach the dispensing desks while walking briskly, return, and then set up and operate the oxygen tanks was determined to be two minutes in pilot studies by the investigators (TTL and ELS). This baseline of two minutes was set as the minimum time in which the experiment could be completed. Clinical staff at the dispensing desks were alerted to the experiment so as to not impede delivery of the oxygen tank to the test subjects. Once ten students were tested from each clinic, the experiment was moved to another one of the four clinical areas (Figure 1).
All students, prior to working on patients at the beginning of the third year, are required to participate in clinic orientation sessions. These training sessions include the locations and use of oxygen tanks for each clinic. Due to the physical layout of this dental school and/or unrelated preclinical training memories, several subjects attempted to obtain portable oxygen tanks from the oral surgery clinic. For the purpose of this study, the oral surgery clinic was considered out of bounds. Students who headed in the direction of oral surgery were redirected to the centralized dispensing area. Subsequently, their times were penalized by adding two minutes to their overall scores. This penalty was determined to be two minutes in pilot studies by two of the investigators (TTL and ELS) as the minimal time required to proceed to the oral surgery clinic and to establish that the required materials were unavailable.

The dispensing desks were equipped with a portable "E" size oxygen tank with an attached oxygen mask. This tank has an outflow valve to release compressed oxygen and a regulator valve to decrease the internal tank pressure down to a working pressure. A gauge allows the user to observe how much gas remains in the tank, which when full holds approximately 680 liters of oxygen (2,200 pounds per square inch). ${ }^{6}$ This size tank can deliver forty-five minutes of oxygen at a flow rate of $15 \mathrm{~L} / \mathrm{min}^{6}$

The data were analyzed using statistical database software (SPSS version 15.1; SPSS Inc., Chicago, IL). Descriptive statistics were computed for each of the study variables. Significance of binary parameters was measured using Fisher's exact test. Timed parameters were compared to the predetermined time using the one-sample t-test. Significance between junior and senior subjects' performance times was determined using the Mann-WhitneyWilcoxon test. An overall $P=0.05$ was considered statistically significant for comparisons.

\section{Results}

We recruited nineteen female and twenty-one male subjects with equal distribution between the D3 and D4 classes, for a total of forty subjects. This group represented 18 percent of the total of D3 and D4 students, which constituted the potentially available study population.

In response to Card \#1 (description of the emergency scenario, Table 1), the subject was asked to provide a free-form verbal outline of his or her 


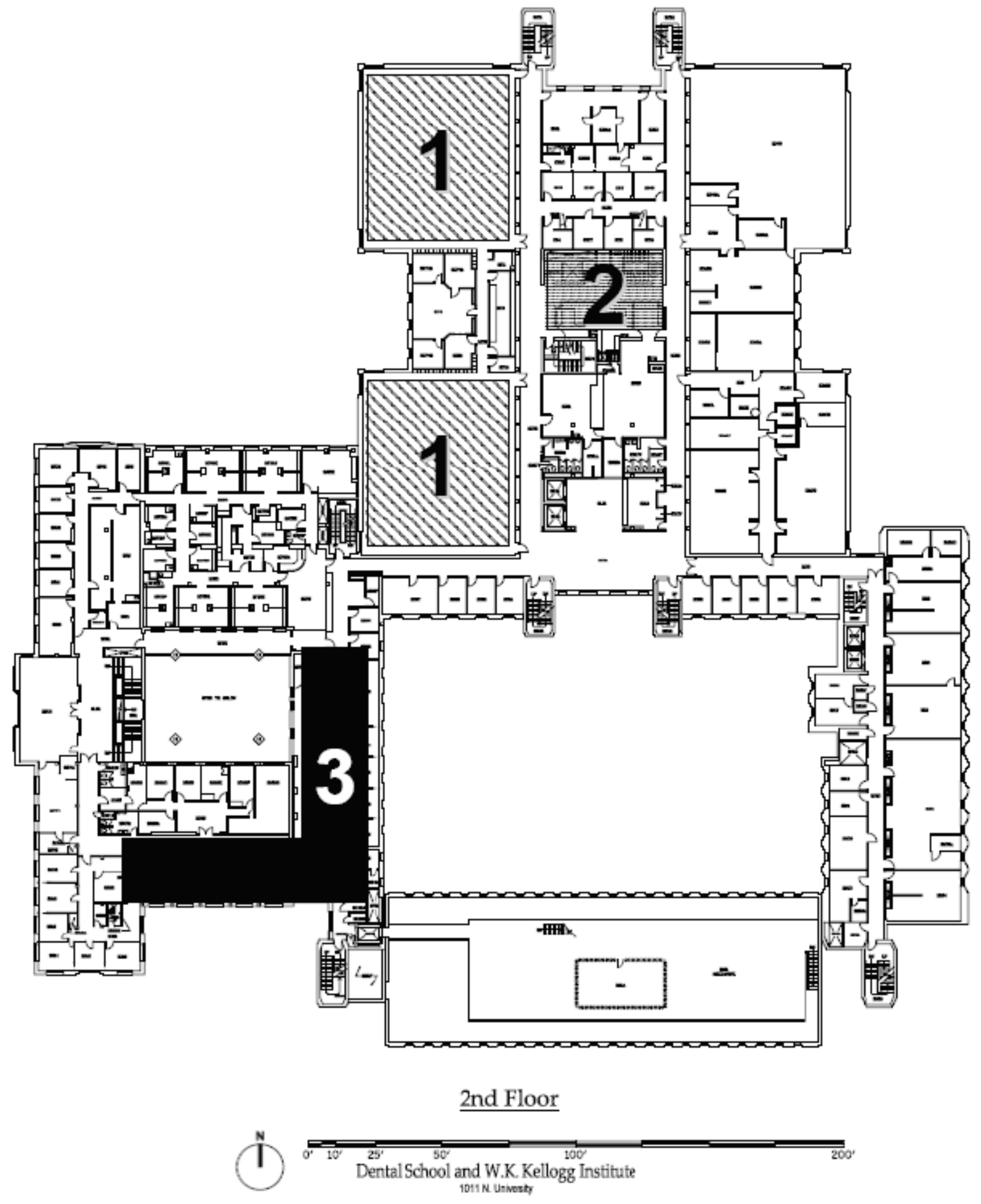

Figure 1. A diagram of the second floor of the University of Michigan School of Dentistry

Note: Downward diagonal shaded areas (1) indicate the undergraduate patient clinics where the experiment was conducted. The closest oxygen tank is located in the narrow horizontal shaded area (2), which is the dispensing location for dental instruments and materials. Oxygen tanks are also located in the outpatient oral surgery clinic, outlined in black (3). There are two additional undergraduate patient clinics on the third floor, located directly above the diagonal shaded areas (1) outlined in the diagram, that follow an identical design with the exception of the oral surgery department. 


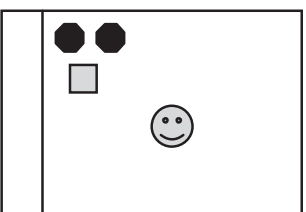

1) Subject is given Card \#1 to read and is observed for the time taken to verbalize the oxygen requirement. This time is recorded $(A)$, and the timer is reset to zero.

Investigators

Subject

Oxygen Tank

(;) Mannequin

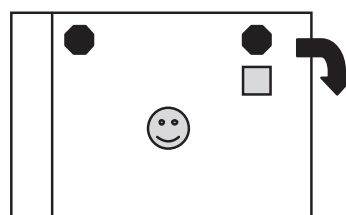

2) Subject is then given Card \#2; timer is started when the student leaves the cubicle. One investigator follows the student to monitor his or her destination.

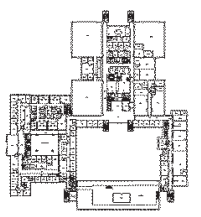

3) Investigator following the student quietly observes his or her ability to locate the oxygen; the student is allowed to ask others for assistance.
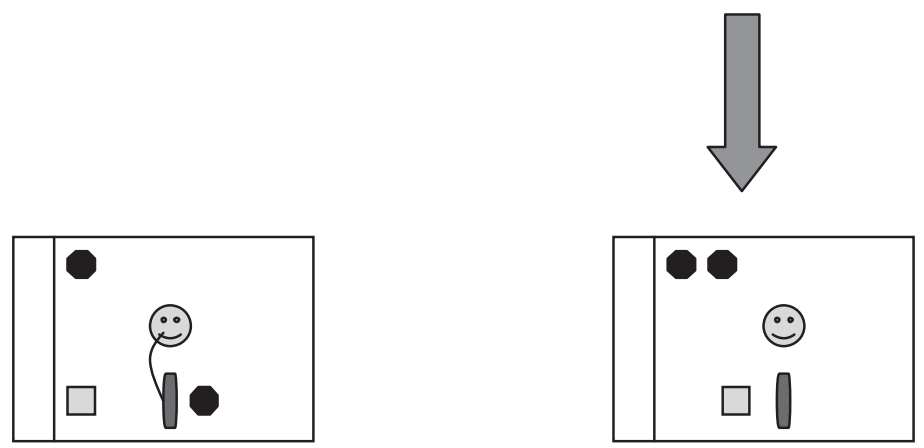

5) Subject has placed the mask on the mannequin, finished operating all valves on the tank, and declares he or she is finished. Final time is recorded by the investigators (C);

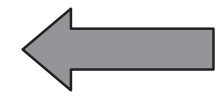

4) Subject returns to the cubicle with the $\mathrm{O}_{2}$ tank; time taken to retrieve the tank is recorded (B). Student proceeds to operate the tank to administer oxygen. the student fills out the postexperimental survey, and the investigators quietly record the flow rate.

Figure 2. Diagram representing the experimental outline: flow of the study conducted in the four undergraduate dental clinics

Note: A mannequin head was set up in a dental chair in one of the unoccupied dental cubicles. Subjects were provided with Card \#1 and asked to describe their plan to address the emergency situation. A timer documented the length of time it took for subjects to verbalize the need to deliver oxygen. Once the subject stated the need to provide emergency oxygen, the timer was stopped and he or she was given Card \#2. The timer was restarted when the subject and investigator left the cubicle to retrieve the oxygen tank. The time to return to the mannequin was recorded as was the time necessary to operate the valves on the tank, connect the mask to the tank, and place the mask on the mannequin. When the subjects believed they had successfully delivered oxygen to the mannequin, they were asked to state so. This final time, which is the total time taken to find and operate the tank, is shown as the average of each class in Table 4. 
response plan. Seven out of forty subjects stated they would terminate all dental procedures they were providing; nineteen would monitor for vital signs; twenty-five would confirm that nitroglycerine had been taken; eight opted to provide another dose of nitroglycerine after five minutes; twenty-two said they would place the patient in a more comfortable position; nineteen said they would activate emergency medical services; ten said they would recruit assistance from a clinical instructor; and three would provide aspirin for the patient (Table 2). Twelve D3 and fifteen D4 participants identified the need for administration of oxygen, representing an overall total of 68 percent (Table 3). Thirty percent of the subjects tested (12/40) were unable to determine that oxygen was required and had to be prompted to retrieve the tank (Table 3). Only 68 percent knew the proper location of the nearest oxygen tank (Table 3 ).

On average, fifty-two and forty-two seconds elapsed before the D3 and D4 subjects, respectively, verbalized that they would deliver oxygen to the patient (Table 4). On average, the subjects required approximately two minutes just to return to the cubicle (Table 4). An additional one minute and twelve seconds on average were required to operate the tank before the scenario was completed. Only 15 percent $(6 / 40)$ were able to perform the entire exercise within the predetermined two-minute optimal execution time for the clinical scenario (Table 3).

Upon completion of the simulated medical emergency, subjects were asked to complete a short written survey. During this time the investigators recorded the flow rate of oxygen set by the subjects. Subjects were not aware that this was also a test variable of the study. A summary of all these values is given in Figure 3. All students opened the outflow valve, but one-half neglected to adjust the flow rate, thus prohibiting oxygen flow to the mask. The suggested flow rate for this scenario was $6 \mathrm{~L} / \mathrm{min}$; seven students (18 percent) were successful in choosing the correct flow rate.

Table 2. D3 and D4 students who were able to perform the actions, by percentage of total subjects and number who carried out the specified procedure

\begin{tabular}{lcrr} 
Action & D3 $(\mathrm{N})$ & $\mathrm{D} 4(\mathrm{~N})$ & Overall $(\mathrm{N})$ \\
\hline Elects to monitor blood pressure & $40 \%(8)$ & $55 \%(11)$ & $48 \%(19)$ \\
Places patient in the cardiac position & $65 \%(13)$ & $45 \%(9)$ & $55 \%(22)$ \\
Notifies an instructor & $30 \%(6)$ & $20 \%(4)$ & $25 \%(10)$ \\
Stops the dental procedure & $20 \%(4)$ & $15 \%(3)$ & $18 \%(7)$ \\
Confirms the patient's medication & $75 \%(15)$ & $50 \%(10)$ & $63 \%(25)$ \\
Elects to give additional nitroglycerine & $20 \%(4)$ & $20 \%(4)$ & $20 \%(8)$ \\
Activates emergency medical services (EMS) & $40 \%(8)$ & $55 \%(11)$ & $48 \%(19)$ \\
Provides aspirin & $5 \%(1)$ & $10 \%(2)$ & $8 \%(3)$
\end{tabular}

Table 3. D3 and D4 students able to identify the oxygen requirement and locate the tank, by percentage of total subjects and number

\begin{tabular}{llll} 
Action & D3 $(\mathrm{N})$ & $\mathrm{D} 4(\mathrm{~N})$ & Overall $(\mathrm{N})$ \\
\hline Identify need (no prompt required) & $60 \%(12)$ & $75 \%(15)$ & $68 \%(27)$ \\
Not able to identify need independently & $40 \%(8)$ & $20 \%(4)$ & $30 \%(12)$ \\
Identify location (no assistance required) & $65 \%(13)$ & $70 \%(14)$ & $68 \%(27)$ \\
Complete experiment in two minutes & $10 \%(2)$ & $20 \%(4)$ & $15 \%(6)$
\end{tabular}

Table 4. Average times for D3 and D4 students to perform the specified tasks (time in mean minutes \pm standard deviation

Action

Identify need for $\mathrm{O}_{2}$

Return to cubicle

Acquire and operate tank
D3

$0: 52 \pm 0: 40$
$2: 17 \pm 1: 28$
$3: 41 \pm 1: 50$
D4

$0: 42 \pm 0: 40$

$2: 05 \pm 1: 24$

$3: 16 \pm 1: 32$ 


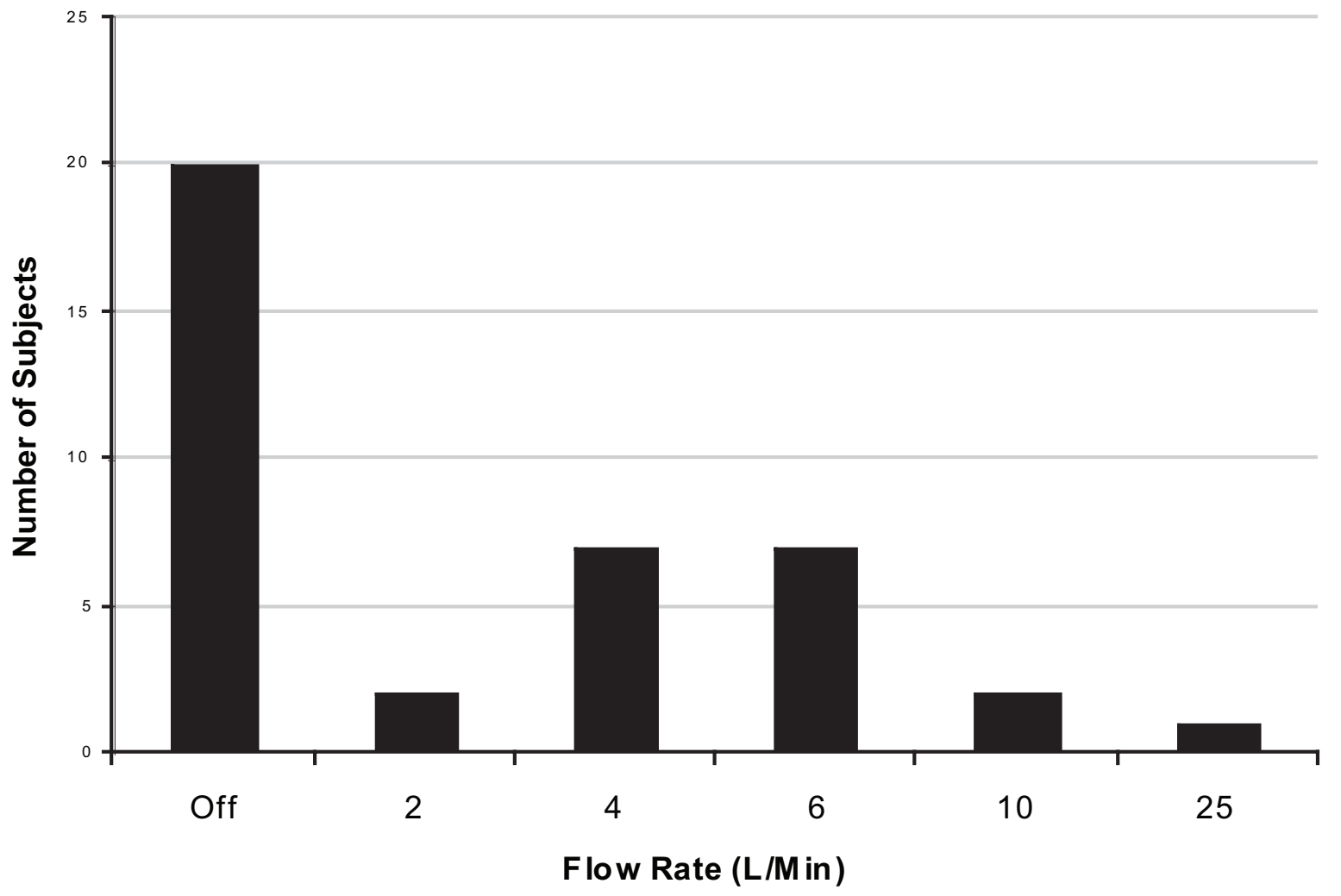

Figure 3. Flow rate of oxygen selected by subjects $(\mathrm{N}=40)$

\section{Discussion}

Our specific aim in this study was to examine the ability of dental students to properly administer oxygen to a simulated patient. Under the conditions of our study, only 68 percent of the subjects identified the need to administer supplemental oxygen to the simulated patient, and only 68 percent of the subjects knew the location of the oxygen tanks. While all students opened the outflow valve, 50 percent neglected to adjust the flow rate, thus prohibiting oxygen flow to the mask.

Second-year dental students participate in a didactic course pertaining to the causes of medical emergencies, the common drugs used, and the protocols followed to manage each emergency situation at the University of Michigan School of Dentistry. Our study used an emergency scenario in which a patient was experiencing a sudden angina attack. Angina is pain resulting from an inadequate supply of oxygen to the heart muscle. ${ }^{4}$ Most often angina occurs when patients have preexisting coronary artery disease and the person is under physical or emotional stress. ${ }^{4}$ When patients experience angina, our clinical protocols include the termination of all dental procedures, immediate administration of nitroglycerine and oxygen, and subsequent administration of a second dose of nitroglycerine after five minutes if symptoms do not subside. Students are advised to record and monitor vital signs (blood pressure, heart rate, and respiration rate) of the patient. Monitoring of vital signs should continue to be assessed at fiveminute intervals until the situation becomes stable. It is recommended that patients be asked to seek a position that is most comfortable, which is often sitting forward. Supplemental oxygen is to be provided on average at a rate of six liters per minute $(\mathrm{L} / \mathrm{min})$. Since the early symptoms of a myocardial infarction are similar to that of angina, it is also recommended to administer aspirin as a preventative measure for nonallergic individuals. Although there is not a set standard minimum time during which supplemental 
oxygen should be administered, it is widely recognized that immediate administration is beneficial to the patient. Supplemental oxygen aids cardiac function, relaxes the patient by treating the pain, and may prevent fibrillation, myocardial infarction, and cardiac arrest. ${ }^{12-14}$ Oxygen is also recommended for compromised patients with chronic obstructive pulmonary diseases because studies have shown that the short period of higher oxygen saturation will not result in adverse ventilatory depression of the patient. ${ }^{15,16}$

One surprising aspect identified during the course of the investigation was that this was the very first time many students had physically operated the supplemental oxygen equipment. Most had only received training in nitrous oxide analgesia during their second year and had not yet interacted with an actual patient requiring nitrous oxide/oxygen during a dental procedure. This nitrous oxide analgesia training occurred in the oral surgery clinic, possibly explaining why some students' initial reaction was to go to the oral surgery clinic. Additionally, we found that it took subjects forty to fifty seconds to identify and verbalize the need to administer oxygen. While this may not appear to be a long time, the subjects were also explaining the other precautionary steps that would be taken. Although the number of subjects who verbalized each response was relatively low and there were wide variations among each action that was described (e.g., placement in a supine position, administration of nitroglycerine, activation of emergency medical services), there were also differences noted in the order that the responses were verbalized. Discerning procedure sequence order was not the purpose of our investigation, but warrants consideration in future investigations.

An area of great concern was the large number of subjects who failed to correctly operate the regulator of the oxygen tank. Half of the subjects did not adjust the flow rate, which in this case would prevent any oxygen from reaching their patient. Seven subjects set the flow rate to $4 \mathrm{~L} / \mathrm{min}$, and two set the rate at $2 \mathrm{~L} / \mathrm{min}$. While this likely would provide the necessary volume of supplemental oxygen required for the patient, a rate less than $5 \mathrm{~L} / \mathrm{min}$ would cause a patient to re-breathe exhaled carbon dioxide retained within the mask. One subject set the flow rate to $25 \mathrm{~L} / \mathrm{min}$, a rate that might cause additional stress for a conscious patient.

One difficulty in determining the success of students in effectively managing an emergency medical situation was the difficulty in preventing discussion amongst potential participants. Although the study subjects were asked to keep the nature of the experiment to themselves to prevent bias, several participants were overheard discussing their performance with nonparticipants outside of the clinics. As a result, over the course of the study, there was a mild increase, though not significant, in the number of students who correctly identified the need for oxygen. However, the overall time performance between subjects recruited early versus late in the study was not significant (data not presented), possibly due to the fact that the primary outcome for the study was not revealed.

During the past year, there were approximately 44,000 patient visits to the University of Michigan School of Dentistry. In 2006, there were nine emergency events (out of thirteen) that required the administration of supplemental oxygen to the patient. Even though this number is small in comparison to the total number of annual patient visits ( 0.02 percent), it is clearly necessary that every provider promptly recognize and effectively manage these situations. Overall, the participants in the study were surprised that the experiment required them to perform an actual simulation with the oxygen tank, and this unexpected factor was one we sought to maintain. It is almost certain that every practitioner will face a clinical situation similar to this one in the future, with the victim being a patient, staff member, or family member. Therefore, based upon these results, we have reevaluated our assumption that students are competent in managing a similar medical emergency without periodic hands-on simulation exercises as part of the curriculum. Our institution already has a well-developed standardized patient program; it is now evident that a medical emergency standardized patient exercise should be advocated within this program. Simulation-based exercises have been successfully utilized to evaluate medical students for many years, and we believe that this study can be used as a pioneering assessment tool in the field of dental education. ${ }^{17}$

Viewed collectively, these results suggest a possible disconnect between classroom instruction and the clinical setting. Clearly, setting and adhering to a set of well-defined standards are challenges for any large institution. Unfortunately, variation in the design and location of the existing oxygen tanks and emergency equipment can place patients at risk. One unexpected aspect of this study that was identified at our institution was a number of irregularities among the thirteen emergency oxygen tanks distributed 
throughout our building. Although the equipment is checked regularly for function and oxygen levels, the investigators identified that there were six different types of oxygen regulators attached to the thirteen oxygen tanks around the school. A lack of standardization of the emergency oxygen equipment complicates training and may place patients at risk. Likewise, it was frequently noted that the oxygen masks or nasal cannulas were not always attached directly to the tank. In some cases, these materials were located in other parts of the clinic or were stored in a location that was not readily accessible. Often the oxygen tanks themselves were located in inconspicuous areas or had been moved by individuals who neglected to inform the rest of the staff.

As an immediate direct result of this study, the following four changes have been implemented at the University of Michigan School of Dentistry:

1. Tanks and regulators throughout the school have been standardized by having existing flow regulators removed, and a single new regulator was purchased and fitted to all existing oxygen tanks in the school.

2. It has also been determined that the oxygen tanks will no longer be located at the dispensing desks; additional tanks have been purchased and placed directly in each of the four undergraduate clinics. These tanks are now positioned near high-traffic areas for optimal access to both staff and students.

3. An online training course has been developed showing recommended procedures for managing simple emergencies and is available to all staff, students, and faculty.

4. All oxygen tanks now have simple to operate oxygen masks or nasal cannulas directly attached. No longer are these items housed separately at remote locations.

This study was initiated as part of a capstone project for the University of Michigan's Scholars Program in Dental Leadership. Leadership in dental education has been defined in many contexts. In this case, curriculum leadership for effective learning and teaching was examined in the context of a medical emergency. While designed exclusively as an evaluation study of the ability of students to translate didactic lessons into practice, the study did not directly compare didactic instruction versus simulated instruction. However, our results identified significant local discrepancies and interpretations in emergency procedures. Due to these results and the subsequent actions taken by our institution's admin- istration, it seems reasonable that our students will be better prepared to manage a medical emergency of this nature in the future.

It is our hope that this experiment will serve as a stimulus for other dental institutions to evaluate their educational practices, specifically with regard to oxygen administration training but in other areas as well. The identification of existing problems by the student investigators enrolled in the University of Michigan School of Dentistry Scholars Program for Dental Leadership — and subsequent modifications to rectify these issues by the school leadership - forms the fundamental basis for maintaining high standards of dental education and providing quality leadership training. Moreover, this study demonstrates that leadership can be flexible and willing to examine itself for the betterment of all constituents, including listening to its own students.

\section{Acknowledgments}

The authors would like to extend their sincere thanks to the undergraduate directors, Dr. Donald R. Heys, Dr. Henry J. Temple, Dr. Mark D. Snyder, and Dr. Ronald J. Heys for facilitating the conduct of this study; Dr. Lina N. Karam for support and advice; Mary Garrelts, Coral Adas, Cheryl Quiney, and Norma J. Mcdougall for their assistance in providing equipment and securing the experimental locale; Thuy D. Le, PA-C from the University of Michigan Hospital for his advice on management of the cardiac patient; and the Dispensing Desk staff for their participation. The authors would also like to thank Dr. Tom Green for inspiration and encouragement. This project was supported in part with funds from the University of Michigan's Scholars Program in Dental Leadership.

\section{REFERENCES}

1. Stehr SN, Weber S, Koch T. A statewide survey of medical emergency management in dental practices: incidence of emergencies and training experience. Emerg Med J 2008;25:296-300.

2. Malamed SF. Medical emergencies in the dental office. 5th ed. St. Louis: Mosby, 2000:3-5,39-41.

3. Centers for Disease Control and Prevention. Healthy aging for older adults. At: www.cdc.gov/aging/. Accessed: May 27, 2008.

4. Malamed SF. Emergency medicine: preparation and basics of management. Dent Today 2001;20:64-7.

5. Smith BK. Treating syncope in the dental office. J Indiana Dent Assoc 2001;80:7.

6. Moore PA. The use of oxygen and airway modalities in dental office medical emergencies. Tex Dent J 2004;121: $1150-5$. 
7. Gill DS, Sharma V, Whitbread M. Emergency drugs in dental practice. Dent Update 1998;25:450-60.

8. American Dental Association. Accreditation. At: www. ada.org/prof/ed/accred/index.asp. Accessed: May 27, 2008.

9. American Dental Association, Commission on Dental Accreditation. Accreditation standards for dental education programs. At: www.ada.org/prof/ed/accred/standards/ predoc.pdf. Accessed: May 27, 2008.

10. Henzi D, Davis E, Jasinevicius R, Hendricson W. North American dental students' perspectives about their clinical education. J Dent Educ 2006;70(4):361-77.

11. Chmar JE, Harlow AH, Weaver RG, Valachovic RW. Annual ADEA survey of dental school seniors, 2006 graduating class. J Dent Educ 2007;71(9):1228-53.

12. Haas DA. Emergency drugs. Dent Clin North Am 2002;46(4):815-30.
13. Waters BG. Providing dental treatment for patients with cardiovascular disease. Ontario Dentist 1995;72(6):24 6,28-32.

14. McCarthy FM. A minimum medical emergency kit. Compendium 1994;15(2):214,216,218-20,224.

15. Austin M, Wood-Baker R. Oxygen therapy in the pre-hospital setting for acute exacerbations of chronic obstructive pulmonary disease. Cochrane Database Syst Rev 2006;3: CD005534.

16. Moloney ED, Kiely JL, McNicholas WT. Controlled oxygen therapy and carbon dioxide retention during exacerbations of chronic obstructive pulmonary disease. Lancet 2001;357(9255):526-8.

17. Schwid HA, Rooke GA, Carline J, Steadman RH, Murray WB, Olympio M, et al. Evaluation of anesthesia residents using mannequin-based simulation: a multiinstitutional study. Anesthesiology 2002;97:1434-44. 